\title{
The phenotypic impact of the male-specific region of chromosome-Y in inbred mating: the role of genetic variants and gene duplications in multiple inbred rat strains
}

Jeremy W. Prokop ${ }^{1,2,3^{*}}$, Shirng-Wern Tsaih², Allison B. Faber ${ }^{2,3}$, Shannon Boehme ${ }^{4}$, Adam C. Underwood 5 , Samuel Troyer ${ }^{4}$, Lauren Playl ${ }^{4}$, Amy Milsted ${ }^{4}$, Monte E. Turner ${ }^{4}$, Daniel Ely ${ }^{4}$, Almir S. Martins ${ }^{6}$, Marek Tutaj ${ }^{2}$, Jozef Lazar ${ }^{1,2,3}$, Melinda R. Dwinell ${ }^{2,3}$ and Howard J. Jacob ${ }^{1,2,3}$

\begin{abstract}
Backgound: The male-specific region of chromosome-Y (MSY) contributes to phenotypes outside of testis development and has a high rate of evolution between mammalian species. With a lack of genomic crossover, MSY is one of the few genomic areas under similar variation and evolutionary selection in inbred and outbred animal populations, allowing for an assessment of evolutionary mechanisms to translate between the populations.

Methods: Using next-generation sequencing, MSY consomic strains, molecular characterization, and large-scale phenotyping, we present here regions of MSY that contribute to inbred strain phenotypes.

Results: We have shown that (1) MSY of rat has nine autosomal gene transposition events with strain-specific selection; (2) sequence variants in MSY occur with a 1.98-fold higher number of variants than other chromosomes in seven sequenced rat strains; (3) Sry, the most studied MSY gene, has undergone extensive gene duplications, driving ubiquitous expression not seen in human or mouse; (4) the expression profile of Sry in the rat is driven by the insertion of the Sry2 copy into an intron of the ubiquitously expressed Kdm5d gene in antisense orientation, but due to several loss of function mutations in the Sry2 protein, nuclear localization and transcriptional control are decreased; (5) expression of Sry copies other than Sry2 in the rat overlaps with the expression profile for human SRY; (6) gene duplications and sequence variants (P76T) of Sry can be selected for phenotypes such as high blood pressure and androgen receptor signaling within inbred mating; and most importantly, (7) per chromosome size, MSY contributes to higher strain-specific phenotypic variation relative to all other chromosomes, with 53 phenotypes showing both a male to female and consomic cross significance.
\end{abstract}

Conclusion: The data presented supports a high probability of MSY genetic variation altering a broad range of inbred rat phenotypes.

Keywords: MSY, Rattus norvegicus, Inbred mating, Sry, Med14y, Ube2q2y, Gene duplications, Phenotypic variation

\footnotetext{
* Correspondence: jprokop54@gmail.com

'HudsonAlpha Institute for Biotechnology, 601 Genome Way, Huntsville, AL

35806, USA

${ }^{2}$ Human and Molecular Genetics Center, Medical College of Wisconsin,

Milwaukee, WI 53226, USA

Full list of author information is available at the end of the article
} 


\section{Background}

Recent analysis of the male-specific region of chromosome-Y (MSY) has identified a core set of genes found throughout mammalian evolution [1]. While many of these genes contribute to classical sex determination and testis function in mammals, they additionally contribute to the maintenance of X-Y gene expression levels and numerous sex disparities in diseases $[2,3]$. The association of human age-related loss of MSY with mortality age in males [4], in combination with a ubiquitous expression profile for several human MSY genes [3], signifies functional importance of genes residing on MSY to normal biological functions outside of sex determination. Disease associations for MSY include cardiovascular disorders, asthma, autoimmune disorders, birth defects, neurological/psychiatric disorders (including schizophrenia and Parkinson disease), and many cancers $[3,5]$. Due to the lack of the MSY to undergo recombination, it is one of the largest linkage disequilibrium (LD) blocks within the human genome; therefore, identifying the causal variants from haplotypes is very difficult using human genomics alone, making it as difficult as autosomal variant analysis before the completion of the 1000 Genomes Project. With the complex genetics of the MSY, research into this field has also been slowed by the lack of animal congenic mapping and the lack of high-throughput characterizations in MSY animal consomic models.

However, the high mutation rate within MSY and an identical inheritance pathway between outbred and inbred animals could allow for comparative genomics in animal models to help identify MSY genes that are involved in phenotypes outside of sex determination, and therefore could be used to look for variants in human that contribute to disease. To date, no animal models with both genetics and high-throughput phenotyping have been developed to study these broad phenotypic contributions of MSY genetics.

Sex differences in cardiovascular disease have been shown previously [6]. MSY haplotypes in human correlate with increased risk of coronary artery disease and blood pressure [7, 8]. The rat (Rattus norvegicus) has been a model organism for MSY contributions to blood pressure regulation for 25 years [9], but has lacked MSY sequence information until 2014, making it impossible to determine mechanisms of MSY phenotypic contributions. Consomic rats generated introgressing MSY between common strains of rats by our group and others for SHR to WKY [10], WKY to SHR [10], SHRSP to WKY [11], BN to SHR [12], BN to FHH [13], and BN to SS [14] have suggested strain-specific contributions of the rat MSY to blood pressure and kidney function (Fig. 1 , strain abbreviations can be found in the figure). An interplay between MSY and androgen receptor signaling has been previously suggested for the SHR blood pressure regulation [10]. An F1 cross of the SHR/y (consomic of the SHR MSY onto WKY autosomes) to the testicular feminized rat (Tfm) repressed the role of MSY on blood pressure [10], but no understanding of the mechanisms behind this has yet been discovered.

Due to a high mutation rate of MSY, combined with a lack of chromosomal crossover, it is hypothesized that many inbred rat strains have had genetic variants on MSY selected upon for phenotypic traits such as blood pressure. These genetic variants of rat MSY may influence similar genes as those involved in human disease or MSY evolution throughout speciation, allowing researchers to narrow down the large human haplotype block to individual genes involved in these processes. As the rat commonly serves as a model for understanding sex differences through hormones, it is critical to define the genetics of strain-specific changes that may also serve to alter sex differences, resulting in a fuller understanding of both genetic and hormone contributions to sex differences in our model organisms. Therefore, we have undertaken an analysis of MSY genes in the most commonly researched strains of rat, which have previously had autosomal genomes sequenced [15]. The characterization of genes within rat MSY, variants within multiple inbred lines, molecular characterization of several protein-coding variants, and a large-scale analysis of MSY phenotyping for several strains will contribute to future development of animal models to study the role of MSY genes.

\section{Methods}

RNAseq analysis for Sry and other chromosome-Y genes

Analysis of RNAseq datasets for Sry was initially performed on publically available RNAseq datasets in NCBI for $R$. norvegicus differentiating Sry2 from the other Sry copies. This was performed using the Sequence Read Archive (SRA) Nucleotide BLAST tool of NCBI optimized for megablast using Not_Sry2, Not_Sry2_2, Sry2, and Sry2_2 sequences of Additional file 1: Table S1. All positive reads were $100 \%$ homologous with an $E$-value $<9 \mathrm{e}-12$. Positive reads for each RNAseq experiment were normalized with total number of reads in the SRA file and shown as reads per million (RPM). BLAST analysis was also performed using the Rat BodyMap datasets of the Fischer 344 rat RNAseq for 11 tissues of four ages in both males and females [16]. Additional sequences for Sry copy specificity (Additional file 1: Table S1) were used in the analysis to determine relative expression of each copy. The single-nucleotide variants (SNVs) in these sequences were placed in the middle or on the ends of the sequence such that reads with $100 \%$ homology and an $E$-value $<9 \mathrm{e}-12$ all contained the SNV. One female genome (LE- ERR224452) was used as a negative control for all MSY variant sites. 


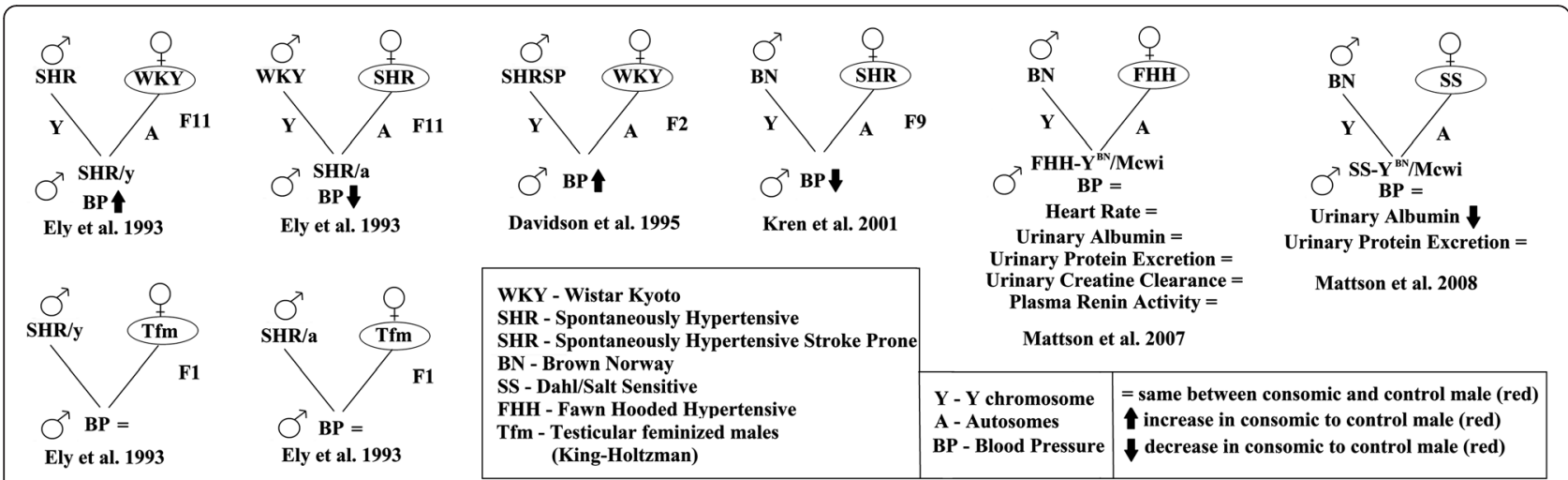

Fig. 1 MSY consomic rats that have been generated. Crosses of male $\left({ }^{\lambda}\right)$ and female ( $(+)$ rat strains have generated six different MSY consomic strains. Abbreviations for rat strains are shown in the first box. Phenotyping of the animal was performed at the designated generation (F11, F2, F9) relative to a male from the strain designated in a circle. For two of the consomic strains, they were crossed with the Tfm rat, containing a loss of function androgen receptor mutation, to assess the segregation of blood pressure contributions due to MSY vs. MSY and hormone signaling

Utilizing the SNVs in the male to female genome analyses below, SNVs were identified that are within 20 bases of each other for the various male-specific SNVs. These sites then had a BLAST analysis done against HTGS files of the rat to confirm they are present on chromosome-Y BAC sequences. Then using these sequences with the SNVs (Additional file 1: Table S1), the 160 male RNAseq files of the Rat BodyMap were analyzed for tissue expression using BLAST with the above parameters.

\section{Analysis of whole-genome sequencing data for chromosome-Y SNVs and indels}

The DNA of seven male rat genomes (ACI, FHH, FHL, $\mathrm{SBH}, \mathrm{SBN}, \mathrm{SR}$, and SS) was sequenced with the Illumina HiSeq2000 platform (Illumina, Inc., San Diego, CA) at the Medical College of Wisconsin. The Rnor_6.0 rat assembly containing the SHR/Akr chromosome-Y was obtained and indexed with BWA (version 0.7.7). Paired-end reads of each male rat genome were aligned to Rnor_6.0 reference sequences with BWA-MEM (version 0.7.7). Read pairing information and flags in the alignment were further cleaned up with fixmate in SAMtools (version 1.1). Duplicates were marked with Picard (version 1.108) prior to variant detection. Variants were called using the Genome Analysis Toolkit (GATK) UnifiedGenotyper (version 3.2.2) with the parameter setting suggested by the best practice (https://www.broadinstitute.org/gatk/guide/ best-practices?bpm=DNAseq).

\section{DNA analysis for chromosome-Y genes in multiple rat strains}

Analysis of Sry copies in genomic DNA reads of eight male rat strains (SHR- ERR224462, WKY- ERR224468, FHH- ERX199109, FHL- ERX199110, ACI- ERX199106, SR- ERX199124, SS- ERX199126, Fischer 344- ERR224448) was performed by BLAST analysis of SRA files using Sry-specific SNVs to differentiate the multiple copies (Additional file 1: Table S1). One female genome (LEERR224452) was used as a negative control.

Whole-genome sequence reads from 20 inbred rat strains (BBDP, LE/Stm, MHS, MNS, WAG, ACI, F344/ NCrl, FHH, FHL, LEW, LEW/NClrBR, LH, LL, LN, SBH, SBN, SHRSP/Gla, SR/Jr, SS/Jr, SS_JrHsdMcwi) were aligned to the rat reference genome Rnor_5.0 (lacking MSY) using BWA, and SNVs were identified in all samples using GATK. The ratio of alternative to reference reads in each strain was calculated from the GATK variant calls to determine zygosity at each locus. With a compiled list of SNVs, we also determined if the genomic DNA sequenced for each of these 20 strains was male (and male contaminated female) or female with BLAST analysis of the reads using the Sry1 coding sequence. In order to identify male-specific SNVs in the whole genome, those genomes that were female were then set to allele frequency of $0 \%$ for SNVs while the male genomes were set to anything between 0.1 and $99.9 \%$. Additionally, we choose to identify strainspecific SNVs in the males again using $0 \%$ allele frequency for the female genomes, setting one male allele frequency at a time to $0 \%$, and setting all other male allele frequency to $0.1-99.9 \%$. For each variant, the average allele frequency was calculated for all male rat strains for each SNV and then averaged over the multiple SNVs located within a single gene, calculating the standard error of the mean (SEM) of the later.

Sry expression using real-time PCR and fragment analysis Male 15-20-week-old rats were anesthetized by intramuscular injection of $2.5 \%$ sodium pentothal at a concentration of $2 \mu \mathrm{L}$ per gram body weight and then terminated by decapitation. Tissues were isolated and 
stored at $-80{ }^{\circ} \mathrm{C}$ until use. RNA was isolated using RNA STAT 60 (Tel-Test Inc, Friendswood, TX) and precipitated with isopropanol. The removal of residual DNA from RNA samples by DNase was carried out using TURBO DNA-free DNase enzyme (Ambion, Waltham, MA). RNA concentration and quality was determined with a Nanodrop ND-1000 Spectrophotometer. Reverse transcription of RNA was performed with ArrayScript Reverse Transcriptase (Ambion) and RtallS-A primer (5' - GGACAGTAAGTAGGTTAGCT-3') that is Sry and strand specific. Real-time PCR was performed with SYBR Green (Applied Biosystems, Waltham, MA) using Sry L (5'-GCG CCC CAT GAA TGC AT-3') and Sry R (5'-TGG GAT TCT GTT GAG CCA ACT-3') primer set on an ABI Prism 7700 Real-Time PCR System. $\triangle C T$ values were analyzed via two-way ANOVA, tissue by strain with age and rat number as random effects, followed by Tukey post hoc test. Differences were considered significant at $P<0.05$. Fragment analysis was performed as previously described [17]. In short, using the cDNA of above following by PCR reactions with GoTaq Flexi DNA Polymerase (Promega, Fitchburg, WI) with 5'S2L (5'-CCA TCT CTG ACT TCC TGG TTG$3^{\prime}$ ) and RtallS-B (5'-AGT AGG TTA GCT GCT GCT AG-3') primers. The amplicon was then labeled with three separate PCR reactions to differentiate the copies using NED-*P1mod(5' -GAA TGC ATT TAT GGT GTG GTC CCG-3') with S1502G1rev(5'-TAG TGG AAC TGG TGC TGC TG-3'), dCAP-Sry 1 HindIII(5'-AGA ATT CAG AGA TCA GCA AGC T-3') with S1502G1rev*-VIC(5' -TAG TGG AAC TGG TGC TGC TG-3'), and 5'S2L with M1*-FAM(5'-TTT GTT GAG GCA ACT TCA CGC TGC-3'). The dCAP reaction was digested with Hinfl restriction enzyme. Samples were run on an Applied Biosystems 3130xl Genetic Analyzer using $5.5 \%$ v/v GeneScan LIZ 600 sizing standard (Applied Biosystems). Data was interpreted with GeneMapper version 4.0.

\section{SRY2 luciferase and cellular localization studies}

The Sry responsive luciferase reporter, pGL3/AR600, was produced by amplifying and inserting 590 bases of 5' UTR from rat androgen receptor, isolated from a single ${ }^{\prime} \mathrm{SHR} / \mathrm{y}$ rat with primers 5'-GTA CCA TGG TTT AGC TTG TCT CTA GCT TCC ACC-3' and 5'CAC CCG GGT AAC TCC CTT TGG CTG A-3'. Amplicons were cleaved using endonucleases $N c o$ I and SmaI, and the resulting restriction fragments were then gel extracted and inserted into pGL3 vector (Promega) opened with the same enzymes. Assembly of all native pEF1/Sry1, 2 , and 3 effector constructs and truncated or site specifically mutated effector constructs was generated.

Chinese hamster ovary $(\mathrm{CHO})$ cells were cultured at $37^{\circ} \mathrm{C}$ in HAM's F12K medium (Sigma-Aldrich, St. Louis,
MO) supplemented with $10 \mathrm{mM}$ HEPES and $10 \%$ FBS (Atlanta Biologicals) in a humidified atmosphere with $5 \% \mathrm{CO}_{2}$. Prior to cotransfection, cells were seeded to 24-well cassettes $\left(6.6 \times 10^{3}\right.$ cells $\left./ \mathrm{cm}^{2}\right)$ and incubated for $16 \mathrm{~h}$. Each well was transiently cotransfected with $50 \mathrm{ng}$ effector plasmid, $500 \mathrm{ng}$ firefly luciferase reporter (pGL/ AR600), and $500 \mathrm{pg}$ of control construct, phRL-null Renilla (Promega), using SuperFect transfection reagent (Qiagen, Venlo, Netherlands) following the manufacture's protocol. After $24 \mathrm{~h}$ incubation, $\mathrm{CHO}$ cells were processed for luciferase activity using the reagents and protocol provided in the Dual-Luciferase ${ }^{\circ}$ Reporter $\left(\mathrm{DRL}^{\mathrm{Tn}}\right)$ Assay System (Promega). Luciferase to Renilla ratios were obtained from measurements collected on a Turner Biosystems 20/20 luminometer and were used to calculate pGL3/AR600 reporter activity of each Sry effector construct relative to reporter activity obtained from $\mathrm{CHO}$ transfected with an $\mathrm{pEF} 1 / \mathrm{Myc} \mathrm{C}$ vector containing no insert. Data reported represent means \pm SEM of three trials conducted in triplicate with each Sry effector construct. Statistical analysis was performed by using one-way ANOVA and a post hoc StudentNewman-Kuels test and Student's $t$ test where applicable. Analyses were run on SigmaStat software (Jandel Scientific, San Rafael, CA) with significance assumed at $P<0.05$.

Various Sry constructs were created from Sry1 and Sry2 pEF1 protein expression vectors to compare regions that differ between Sry1, Sry2, and Sry3 proteins. These constructs were Sry1(HMGbox)-Sry1 with only the HMGbox, Sry1(delPolyQ)-Sry1 glutamine-rich region converted to that of Sry2, Sry2(-QR)-removal of the entire glutamine-rich region, Sry1(20-22AAA)-alanine mutations to the N-terminal nuclear localization motif of Sry1, Sry1(78-79AA) - alanine mutations to the C-terminal nuclear localization motif of Sry1, Sry1(NoNLS)-alanine mutations to both the $\mathrm{N}$ - and C-terminal nuclear localization motifs of Sry1, Sry1(H21R) - histidine to arginine mutation corresponding to the site seen in Sry2, and Sry2(H21R)-histidine to arginine mutation corresponding to the site seen in Sry1. CHO cells grown to approximately $1 \times 105$ cells $/ \mathrm{cm}^{2}$ were transfected with $7.5 \mu \mathrm{g}$ of each respective plasmid DNA using ExGen500 transfection reagent (Fermentas), incubated for $24 \mathrm{~h}$, trypsinized/pelleted, and cytoplasmic/nuclear extracts were prepared using ProteoJET Cytoplasmic and Nuclear Protein Extraction Kit (Fermentas). Cytoplasmic and nuclear protein extracts $(20 \mu \mathrm{g})$ were separated on $13.5 \%$ polyacrylamide gels. Proteins were transferred to PVDF membranes that were blocked for $1 \mathrm{~h}$ at room temperature in PBS containing $5 \%$ nonfat dry milk and $0.1 \%$ Tween-20. SRY proteins where detected using a goat anti-mouse SRY (Santa Cruz Biotechnology, Inc., Dallas, TX) or a goat anti-Myc epitope (Bethyl Laboratories, Montgomery, TX) antibodies, 
diluted in a blocking solution at 1:300 and 1:1000, respectively. After a 1 -h incubation at $22{ }^{\circ} \mathrm{C}$, blots were washed in PBS, following a 1-h incubation with a donkey anti-goat HRP conjugate (Bethyl Laboratories) diluted to 1:3000 in blocking solution. Bands were detected using SuperSignal West Pico Chemiluminescent substrate (Thermo Fisher Scientific Inc.) and visualized with a Kodak 2200 Gel Logic Imaging system. All assays included a control lane containing cell extracts obtained from cells transfected with an expression vector containing no insert.

\section{Protein modeling}

Models for the nonHMGSry protein were generated using the ab initio modeling server Quark [18]. Each of the top five models were run for 10 ns of molecular dynamic simulations using YASARA with the Amber03 force field [19], $0.997 \mathrm{~g} / \mathrm{mL}$ water, $\mathrm{p} K \mathrm{a}$ of 7.4 , and mass fraction of $0.9 \%$. The $Z$-score, wrong isomers, and cis-peptide bonds were calculated using the YASARA2 force field. Combining these calculations with the analysis of movement throughout the molecular dynamic simulations, the models were ranked to determine the most likely structure. The nonHMGSry protein sequence was also analyzed for functional motifs using ELM [20].

\section{SRY-AR synergistic regulation assay}

Luciferase assays on the Sry1 and AR600 promoters were performed and analyzed as previously published [21]. The SRY/AR synergistic regulation experiments were performed using charcoal stripped fetal bovine serum (Innovative Research, Novi, MI) with or without the addition of $100 \mathrm{nM}$ testosterone (Sigma-Aldrich). The hSRY P131T corresponds to the variation seen in SRY1 (P76) to SRY3 (T76) [22]. Androgen receptor (AR) expression vector was purchased from ATCC (vector \#80005, hARa [CL7a-AR 160-910]). For each transfection, $100 \mathrm{ng}$ of expression vector was used per well with single protein expressions using $50 \mathrm{ng}$ of vector (hSRY, $\mathrm{AR}$, or P73T) with $50 \mathrm{ng}$ of empty vector and double transfections (hSRY/P131T with AR) using $50 \mathrm{ng}$ of each. All constructs were sequence confirmed on an ABI 3130xl using BigDye Terminator v3.1.

\section{Sry3 electroporation and blood pressure studies}

A total of ten animals (normotensive WKY rats) were used for three experimental groups. There were three empty vector animals and seven Sry3-treated animals. All of the empty vector animals and four of the Sry3 animals received drug treatment with olmesartan medoxomil. Animals received a standard 12-h light/ dark cycle and were given standard rat chow $(22.5 \%$ protein, $52 \%$ carbohydrate, and $6 \%$ fat by weight,
Prolab 3000, Agway, Syracuse, NY) and water ad libitum. Rats were individually housed in polycarbonate cages $(48 \mathrm{~cm} \times 27 \mathrm{~cm} \times 20 \mathrm{~cm})$ with heat-treated bedding (Sani Chips, R.J.Murphy, Rochelle Park, NJ). Cage changes were performed once a week, scheduled so as not to interfere with blood or urine sampling.

Animals were implanted with an aorta telemetry device (model-PAC40; Data Sciences International, St. Paul, MN), and baseline measurements of systolic pressure, diastolic pressure, heart rate, and activity were collected (RPC-1 and Dataquest A.R.T., Data Sciences International). All animals were monitored for telemetry measurements throughout the study, at 30-min intervals, except for the 24-h periods that animals spent in metabolic cages. Animals were allowed to recover from telemetry surgery for 1 week before a baseline metabolic cage study was done.

All animals were injected and electroporated with PEF1(-) or PEF1/Sry3 $(25 \mu \mathrm{g})$ into the left kidney as previously performed [23], which represents day 1 of the study. On day 6, the first 24-hour urine was taken followed by the first plasma sample on day 8 . On day 14 , animals were given sham- or olmesartan-treated drinking water. The drug was given for 1 week total, and on day 17 , a second 24-h urine was taken followed by a second plasma on day 19. Drug treatment was then stopped and time was given to allow the drug to leave the system. On day 25 , a final $24-\mathrm{h}$ urine was collected followed by a final plasma sample on day 27. All the above animal experiments were approved by the University of Akron IACUC.

Phenotype analysis of BNxFHH and BNxSS consomic panels The chromosome-Y consomic rats were generated as part of the PhysGen Program for Genomic Applications (http://pga.mcw.edu). Detailed phenotyping protocols are posted on the website. Briefly, six phenotyping protocols (Lung, Respiratory, Cardiac, Renal, Vascular, Biochemistry) were run in parallel using ten male and ten female rats per protocol. Rats were studied between 6 and 10 weeks of age and under control conditions or under diet or environmental stress. The consomic rats were studied using the same protocols and quality controls as previously described [24]. Phenotypes listed to significantly differ were determined using adjusted $P$ values $<0.05$ in the consomic strain (for example SS-Y $\mathrm{Y}^{\mathrm{BN}} / \mathrm{Mcwi}$ ) compared to the parental strain (for example the SS). The adjusted $P$ values were calculated by the Mann-Whitney test followed by a Bonferroni adjustment for multiple tests. All animal experiments were approved by the Medical College of Wisconsin IACUC.

\section{Results}

Mammalian conserved protein-coding genes in rat Of mammalian conserved MSY genes, nine (Eif2s3y, Zfy, Usp9y, Ddx3y, Uty, Ube1y, Kdm5d, Rbmy, Sry) are 
confirmed in rat [2] and thus would be primary MSY genes of interest in translatable human disease. All nine conserved genes are expressed in rat testis, with five genes (Eif2s3y, Ddx3y, Uty, Kdm5d, and Sry) expressed in all ten male tissues of the Rat BodyMap project (Table 1). Sry is well known to have undergone gene duplication, with variants defining 11 genes: Sry1, Sry2, Sry3, Sry3A, Sry3B, Sry3BI, Sry3BII, Sry3C, Sry4, Sry4A, and nonHMGSry [21]. Most of these SNPs for individual Sry genes could be mapped onto the rat MSY sequence relative to other human shared genes (Fig. 2a).

\section{Autosomal gene duplications onto the rat MSY}

While identifying Sry copies in the SHR MSY sequence, we found transposable elements were likely causal for duplication of Sry genes and also identified two nonMSY genes, Med14Y and Limd2Y, that have been duplicated from the $\mathrm{X}$ chromosome and autosomes onto the rat MSY [21]. However, the confirmation of these and other duplicated genes onto rat MSY is difficult due to repetitive sequence. To confirm duplicated genes, we developed a novel sequence analysis, comparing variants of genes identified in males but not females, using whole-genome sequencing.

The absolute sex for all reads of 23 sequenced rat genomes was determined, identifying Sry sequence reads in 18 genomes; thus, only 5 genomes are $100 \%$ female DNA (Additional file 1: Table S2). This approach allows for Sry-contaminated female sequences to be treated as males, thus treating SNPs of duplicated genes of males that could also be included with equal probability as Sry sequence. Following alignment of reads to the Rnor 5.0 genome (lacks MSY sequence), all single-nucleotide variants (SNVs) found in protein-coding genes of male genomes and not female genomes identified a subset of genes potentially inserted onto MSY (Table 2). Nine genes are found to have specific SNVs in all 18 male rat genomes with an average allele frequency of $30.7 \%$ over $2790 \mathrm{SNVs}$, close to the $33 \%$ expected frequency for a

Table 1 Conserved $Y$ chromosome genes found in rat

\begin{tabular}{llll}
\hline & Accession number & $\begin{array}{l}\text { Y chromosome } \\
\text { SHR BAC }\end{array}$ & $\begin{array}{l}\text { Primary tissue } \\
\text { expression in rat }\end{array}$ \\
\hline EIF2S3Y & GATN01000003.1 & AC242953.5 & Ubiquitous \\
ZFY & GATN01000010.1 & AC239865.4 & Testis \\
USP9Y & GATN01000001.1 & AC242055.4 & Testis \\
DDX3Y & GATN01000002.1 & AC242055.4 & Ubiquitous \\
UTY & GATN01000009.1 & AC241873.4 & Ubiquitous \\
UBE1Y & EF690356.2 & AC242953.5 & Testis \\
KDM5D & GATN01000004.1 & AC242859.2 & Ubiquitous \\
RBMY & GATN01000006.1 & AC239817.2 & Testis \\
SRY & Multiple & AC239701.6 & Ubiquitous \\
\hline
\end{tabular}

variant found in an autosomal gene that has been duplicated onto the MSY. The duplicated Limd2 was confirmed by this analysis for all strains; however, Med14 was not. Removing individual male genomes from the analysis, we were able to identify strain-specific variants in male duplicated genes, such as the lack of 33 Med14 SNVs in the FHL rat strain, suggesting this gene is not present in the FHL MSY.

Clustered male-specific SNVs from the analysis above (two or more SNVs located within 20 bases), allowing for single-read detection of multiple variants found throughout male rat strains, were used to screen MSY bacterial artificial chromosomes (BACs) and also the Rat BodyMap datasets. Each of the specific SNV sites was detected in SHR MSY sequencing BACs (Table 1), confirming with a secondary sequencing approach the existence of the duplicated genes into the MSY. This identification on MSY BACs also allowed for a prediction of the mechanism of duplication. Seven genes were identified as retroposed (i.e., spliced genes reinserted onto MSY), one as a MSY gene that duplicated to an autosome (RGD1560580) and two as transposed genes (i.e., contains normal introns of gene). Using the Rat BodyMap dataset in combination with the male-specific SNVs, expression was detected for only two retroposed genes, Med14Y (Fig. 2b) and Ube2q2Y (GenBank KM610331, Fig. 2c), in male and not female RNAseq datasets.

$\operatorname{Med} 14 Y$ (Fig. 2b) and Ube2q2Y (Fig. 2c) are expressed in all male but not female tissues of the Rat BodyMap dataset, with their MSY location shown on Fig. 2a. In comparison to Med14Y and Ube2q2Y, Limd $2 Y$ has only three detectable reads out of the 13.3 billion analyzed and also contains one mutation resulting in the deletion of a $\mathrm{Zn}$ coordination site required for structural folding [25] and additional nonsense mutations (Additional file 1: Figure S1). The combination of a lack of transcription and mutations that would inhibit protein function suggests that Limd2Y has become a pseudogene (Limd2Y-ps) on rat MSY in all sequenced male strains. Initial analysis of our rat variant visualizer tool, based on Rnor 3.4 genome alignment lacking MSY sequence, on the rat genome database (http://rgd.mcw.edu/rgdweb/front/ config.html) for all strains showed that these variants for all autosomal genes inserted into the MSY were present at around $1 / 3$ allele frequency in the database. This suggests a possibility of researchers to misidentify MSY gene variants as heterozygous variants in autosomal genes.

To update this information and add ChY gene variants between strains, a new strain genome comparison tool was created. We sequenced male genomes for seven strains (ACI, FHH, FHL, SBH, SBN, SR, SS) and aligned them onto the Rnor 6.0 assembly that 


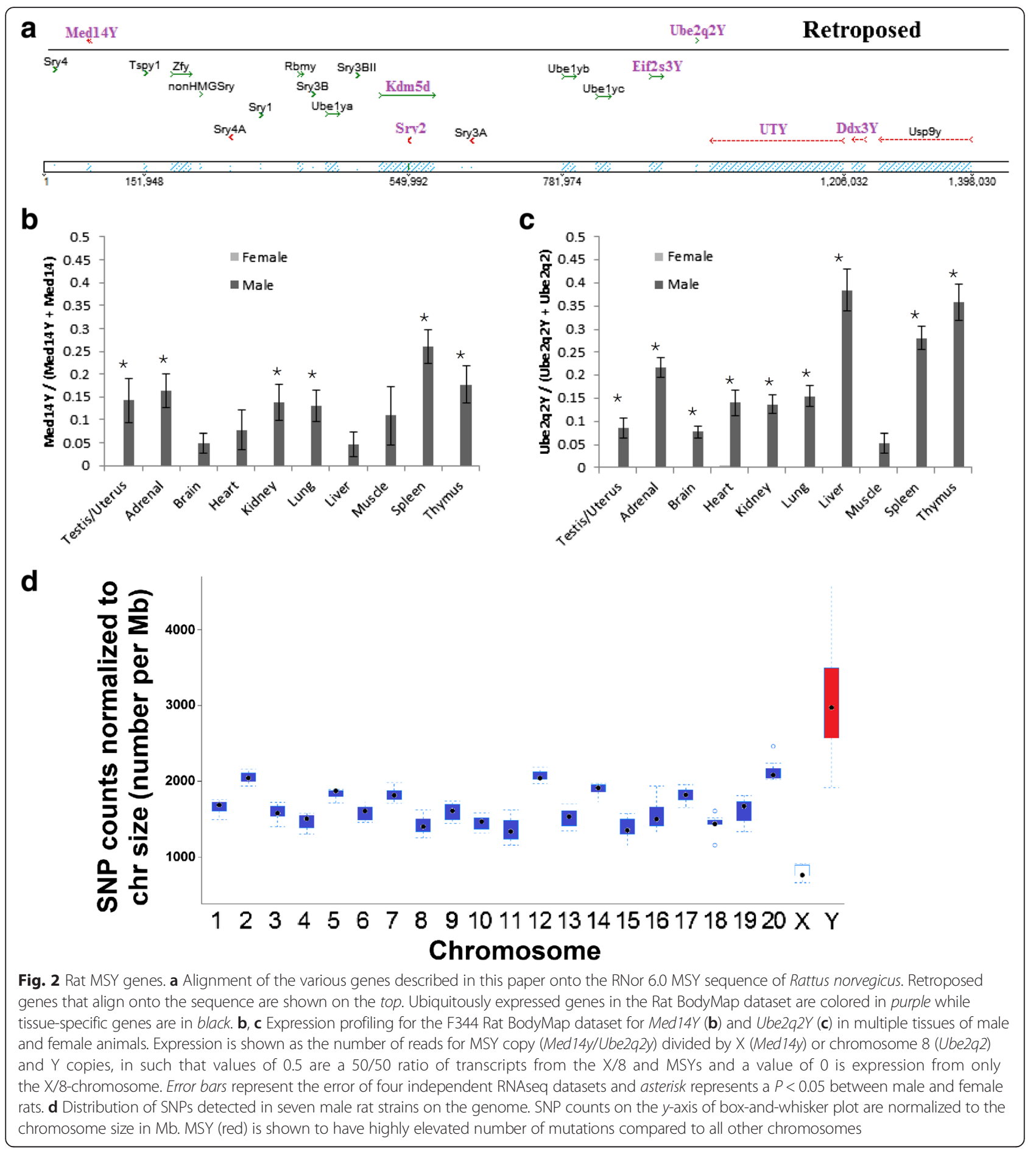

includes newly sequenced MSY. The number of single-nucleotide variants (SNVs) was calculated for each strain and was made relative to total chromosome size (Additional file 1: Table S3). The analysis shows a $1.98 \pm 0.06$-fold elevation of SNVs on MSY relative to all other chromosomes (Fig. 2d), a value that is similar with previous rodent MSY sequencing work [26] confirming MSY wide rate in nearly every analyzed rat strain. To resolve this issue with variant analysis, we have developed and released a new Variant Visualizer from the rat genome database (http:// rgd.mcw.edu/rgdweb/front/config.html?mapKey=360) for variants called from Rnor 6.0 alignment for seven different rat strains. 
Table 2 Autosomal gene duplication to MSY

\begin{tabular}{|c|c|c|c|c|c|c|c|c|c|}
\hline Chromosome & Gene & SNVs & $\begin{array}{l}\text { Average allele } \\
\text { frequency }\end{array}$ & $\begin{array}{l}\text { Strain w/o } \\
\text { SNVs }\end{array}$ & $\begin{array}{l}\text { Strain-specific } \\
\text { SNVs }\end{array}$ & $\begin{array}{l}\text { MSY SHR/Akr } \\
\text { BAC }\end{array}$ & MSY gene & $\begin{array}{l}\text { Duplication } \\
\text { method }\end{array}$ & $\begin{array}{l}\text { Tissue } \\
\text { expression }\end{array}$ \\
\hline chr2 & Ect2 & 25 & $23.9 \pm 2.3$ & - & $\mathrm{SBH}(2), \mathrm{SR}(2), \mathrm{SS}(1)$ & AC241473.5 & Ect2Y_ps & Retroposed & None \\
\hline chr8 & Ube2q2 & 16 & $20.6 \pm 2.1$ & - & $\operatorname{LL}(2)$ & AC241873.4 & Ube2q2Y & Retroposed & Ubiquitous \\
\hline chr10 & Haver2 & 33 & $30.8 \pm 2.3$ & - & - & AC241887.5 & Havcr2Y_ps & Retroposed & None \\
\hline chr10 & Limd2 & 40 & $45.2 \pm 2.9$ & - & $\mathrm{LH}(1), \operatorname{SBN}(1)$ & AC241808.7 & Limd2Y_ps & Retroposed & None \\
\hline chr12 & RGD1560580 & 4 & $24.9 \pm 4.5$ & - & - & AC241548.6 & Ssty2 & MSY duplication & None \\
\hline chr13 & Xpr1 & 24 & $28.9 \pm 2.1$ & - & F344(2), SBN(1) & AC246656.3 & Xpr1_Y1/2/3/4 & Transposed & None \\
\hline chr13 & Prrc2C & 2 & $19.6 \pm 1.8$ & - & - & AC241950.2 & Prrc2cy_ps & Retroposed & None \\
\hline chr14 & Vom2r67 & 5 & $24.4 \pm 2.7$ & - & $\operatorname{SBH}(2), \operatorname{SR}(1)$ & AC242505.8 & Vom2ry_ps & Retroposed & None \\
\hline chr16 & Lsm 1 & 5 & $24.9 \pm 2.2$ & - & - & AC243283.4 & LsmiY_ps & Partial transposed & None \\
\hline $\operatorname{chr} x$ & Med14 & 33 & $31.2 \pm 3.3$ & $\mathrm{FHL}$ & $\mathrm{FHH}(1)$ & AC239701.6 & Med14y & Retroposed & Ubiquitous \\
\hline
\end{tabular}

\section{Sry duplicated genes in commonly used rat strains}

Sry is expressed in numerous human tissues [3]. An overlap of many tissues, such as kidney, is seen for the expression of Sry in primates and rat that are not found in the mouse [27]. This expression is novel to Sry as it is not seen for Sox3, the ChrX homolog of Sry [27]. However, the expression profile of rat Sry is ubiquitous while in human it is not [3]. Unlike the human genome, the rat genome contains 11 functionally distinct copies of Sry (http://bmcgenomics.biomedcentral.com/ articles/10.1186/1471-2164-14-792) likely amplified through gene conversion at repetitive elements [21]. The existence of these 11 copies has only been performed in two strains to date (SHR and WKY), and an expression profile for each copy has never been performed in detail before. Thus, the overlapping function between Sry in rat and human would be better understood by a copy-specific expression analysis in multiple laboratory strains of rats.

The advancing technology of next-generation sequencing allows for the identification of multiple Sry genes at sequence resolution in both genome and transcriptome, further enhancing our capabilities in segregating multiple Sry copies. Utilizing SNVs (Fig. 3a) unique to specific copies of rat Sry (protein shown in Fig. 3b), 8 of 11 Sry copies are confirmed in SHR, WKY, FHH, FHL, SR, SS, and F344 inbred rat strains (Fig. 3c). The remaining Sry3 copy is unable to be differentiated (nd, not determined) from $S r y 3 B I / 3 B I I$ due to short reads used in next-generation sequencing. Our initial work using realtime PCR (Fig. 3d) confirms Sry multiple tissue expression in the rat; however, now that we have established the existence of at least 8 Sry copies in the majority of commonly used rat strains, a more detailed analysis of Sry copies expression is needed.

A fragment analysis protocol was initially developed to identify copy expression specificity, particularly for the
Sry2 gene. Using this approach, Sry2 is observed to have the highest expression of Sry copies in most tissues [17]; however, this approach is limited to the analysis of only a few Sry copies that have variants altering restriction sites. The Rat BodyMap dataset allows, for the first time, the ability to assess expression of each Sry copy in multiple tissues (Fig. 4a). In agreement with our SHR and WKY fragment analysis, Sry2 is the predominantly expressed copy throughout all tissues. To assess Sry expression in other rat strains, we utilized publically available RNAseq datasets in NCBI. Sry transcripts are detected in 197 separate rat RNAseq datasets; however, only 15 of these datasets contain transcripts that are from non-Sry 2 copies (Fig. 4b). This Sry 2 copy, found so ubiquitously transcribed, is located within an intron, in the antisense orientation, of the ubiquitously expressed $K d m 5 d$ gene (Fig. 2a) suggesting the location of the gene insertion has possibly driven elevated expression. Likely to compensate for this elevated expression, SRY2 rapidly accumulated an amino acid mutation (H21R) within the $\mathrm{N}$-terminal nuclear localization site (nNLS) that alters the ability of SRY2 to activate transcription (Fig. 4c) and localize the protein within the nucleus (Fig. 4d, e). Additionally, we have previously shown that SRY2 variants located in the glutamine-rich region (deletion of 13 amino acids corresponding to changes in Sry2 location in SDS-PAGE of Fig. 4d) decrease transcriptional control [21]; however, these mutations and the complete removal of the glutamine-rich region (Fig. 4d, lane 4) do not alter nuclear localization (Fig. 4d), only transcriptional control.

Of the tissues analyzed from the Rat BodyMap and publically available RNAseq datasets, the testis, kidney, lung, spleen, brain, and colon show expression of nonSry 2 copies. Interestingly, the list of rat tissues expressing non-Sry2 genes (all Sry genes excluding Sry2) overlaps nearly perfectly with our previous analysis of the 


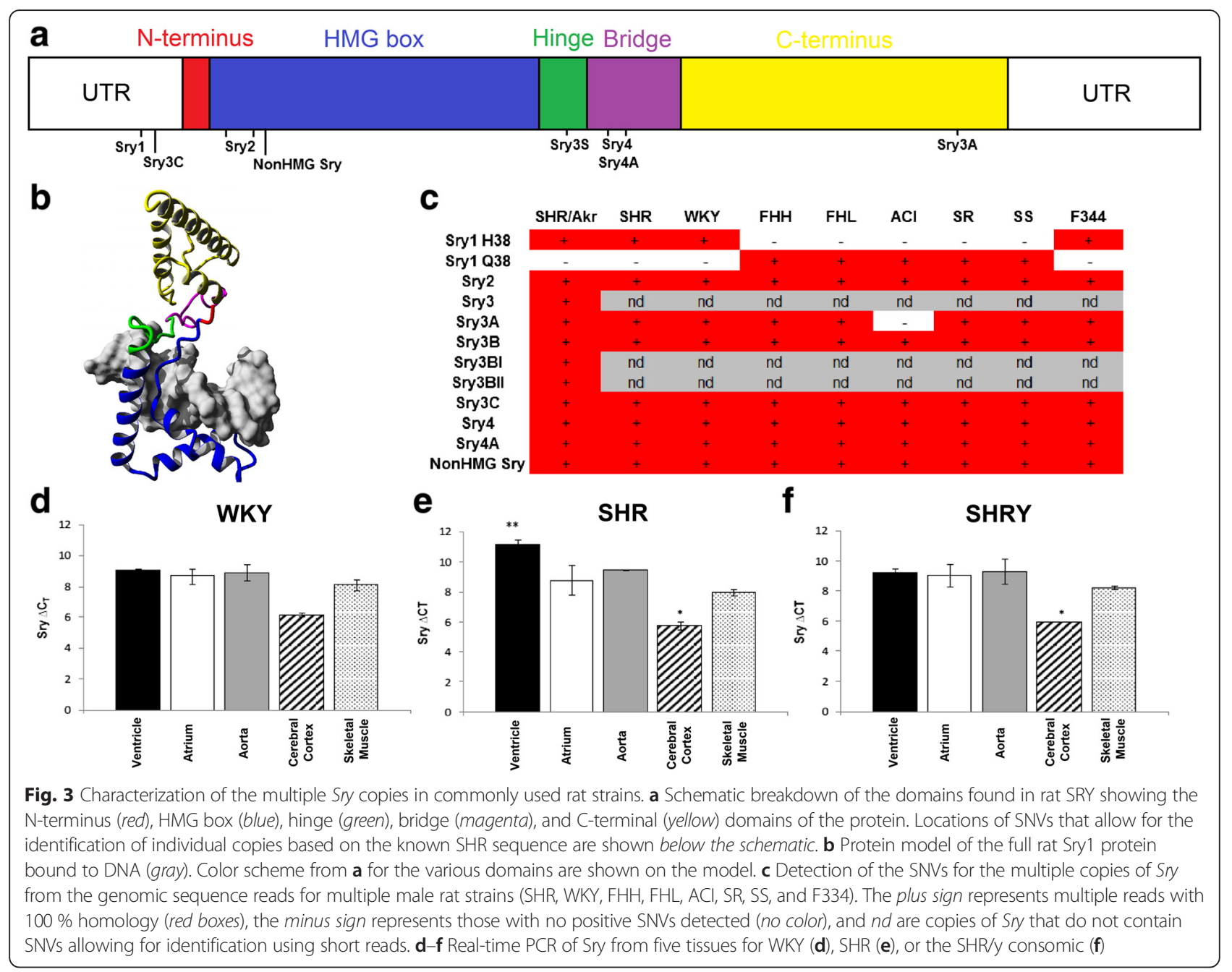

human protein atlas expression profile for human SRY [3]. The lung contains a vast array of Sry copies (Fig. 4a) including the highly conserved nonHMGSry (GenBank KC215141.1) that contains a frame shift mutation directly before the high-mobility group (HMG) domain, but still codes for an open reading frame. This is the first time transcripts from nonHMGSry have been identified. Protein modeling, molecular dynamic simulations, and functional motif analysis of this novel protein elucidate regions of structural order, possible nuclear localization motif, 14-3-3 binding motif, and a degradation box suggesting functional impact for transcripts from this rat conserved gene (Additional file 1: Figure S2).

Mapping of genes in MSY consomic SHR to WKY crosses (Fig. 1) resolved that the SHR rat has a duplication of an Sry3 gene relative to other analyzed strains [28]. The cross of the SHR/y consomic animal to the Tfm rat model (Fig. 1) blocks MSY blood pressure elevation, suggesting that there is interplay between the Sry3 locus and AR signaling. SRY has previously been shown to directly bind AR protein [29]. The SRY3 protein contains an amino acid substitution (proline to threonine) at amino acid 76. Furthermore, we have shown that SRY and $\mathrm{AR}$ can synergistically regulate promoters in a testosterone-dependent manner and that a change from a proline to threonine in SRY results in a loss of this synergistic promoter regulation with AR (Fig. 5a). This threonine point mutation has also been shown to increase regulation of the renin-angiotensin system (RAS) components to elevate the pro-hypertensive angiotensin II peptide in vitro [22] and in vivo [23]. Sry3 copies (Sry3, Sry3A, Sry3B, Sry3BI, Sry3BII) are expressed in rat (Fig. 4a) suggesting that these Sry3 genes may have a kidney function through regulation of the RAS. Delivery of a Sry3 expression vector to the normotensive WKY rat results in a blood pressure elevation that can be blocked with a RAS inhibitor, olmesartan (Fig. 5b). This finding supports Sry3 gene duplication within SHR causing altered testosterone signaling and regulation of the renin-angiotensin system. In light of preliminary data for 


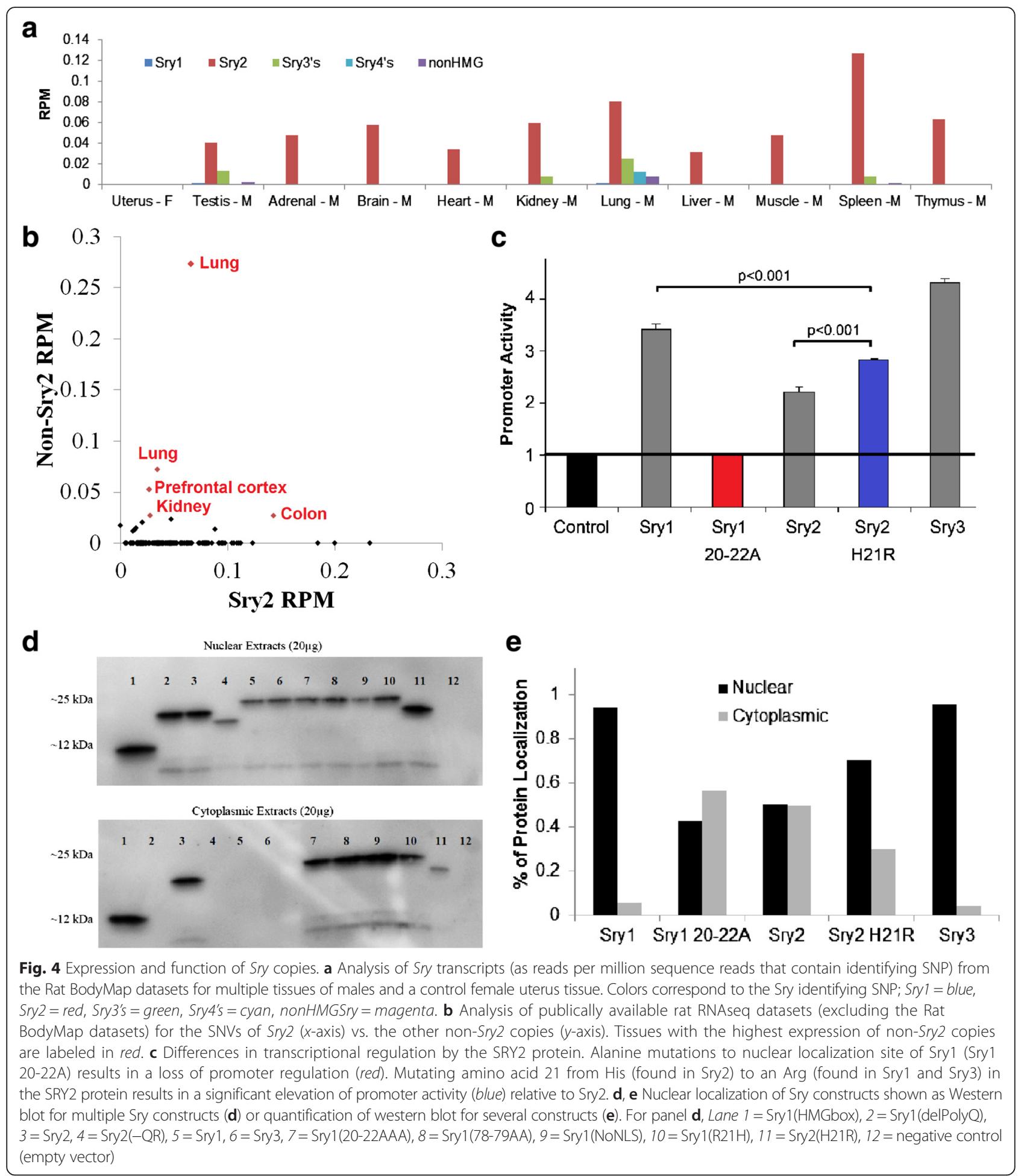

the overexpression of human SRY within the rat kidney, this suggests a high probability of SRY involvement in blood pressure regulation and potentially hypertension, with the rat serving to provide mechanistic understanding of SRY within the kidney.

\section{MSY of other rat strains contributes to phenotypic diversity}

Identifying genes in the MSY that contribute to phenotypes such as blood pressure in the rat suggests the need for a broader phenotyping analysis of MSY contribution. 


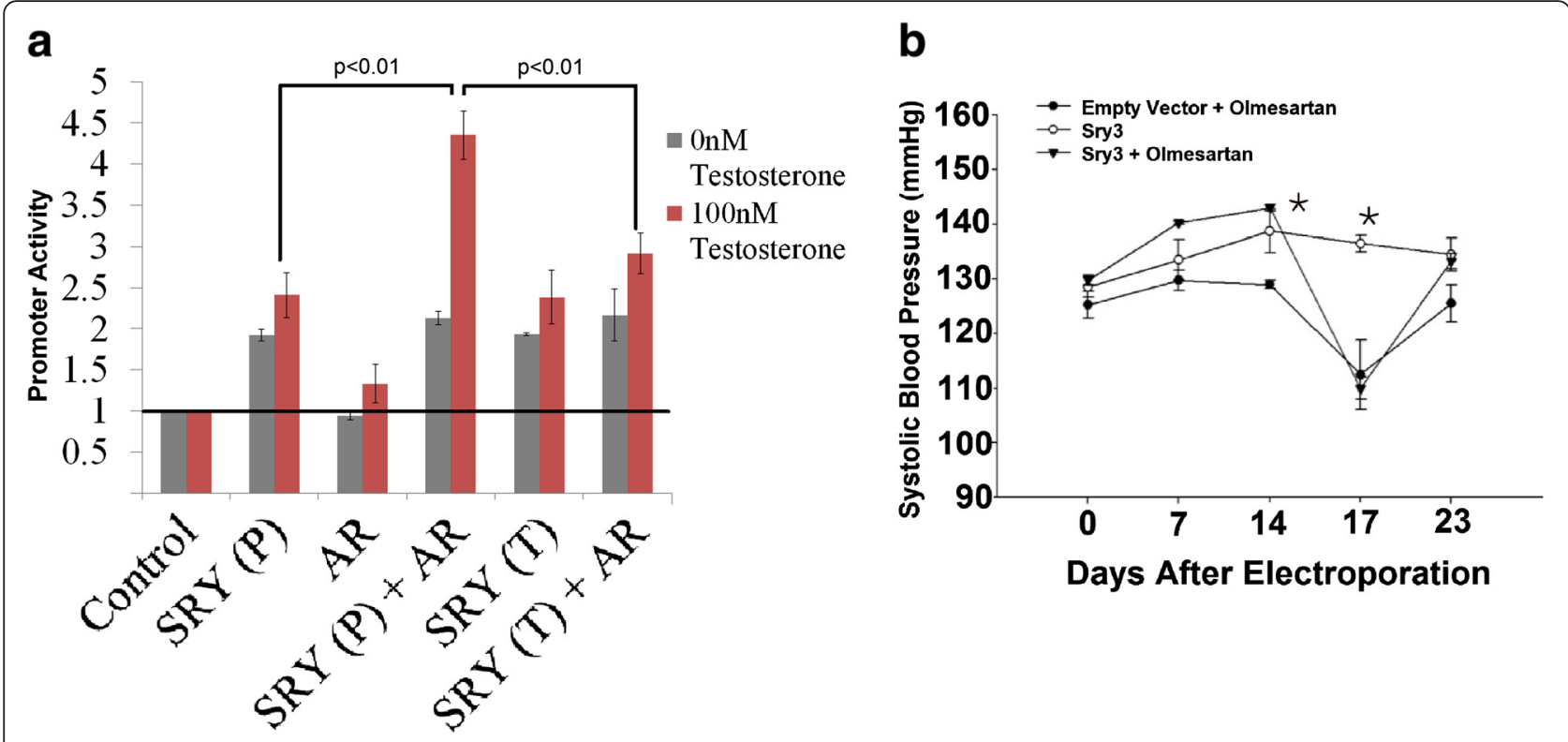

Fig. 5 SRY blood pressure regulation through androgen receptor and the renin-angiotensin system. a Testosterone-dependent synergistic regulation between SRY and AR is altered by mutations to SRY at the location that separates Sry3 (T) and all other rat and mammalian SRY sequences (P). $\mathbf{b}$ Delivery of the Sry3 expression vector (open circle) to the kidney of WKY male rats at day 0 significantly increased blood pressure relative to a control (closed circle) 14 days after vector electroporation. Olmesartan, a RAS inhibitor, administered to control and half of the Sry3-treated animals (closed triangle) at day 14, significantly decreases blood pressure to the same value in both groups. Following removal of olmesartan at day 17 , blood pressure increased more rapidly in the Sry3-treated group (closed triangle). Error bars are shown as the SEM of three to four independent animals with asterisk representing a $P<0.05$ for blood pressure between the Sry 3 and control vector electroporated animals

As part of two large systematic rat consomic panels created and phenotyped by our PhysGen Program for Genomic Applications [13, 14], data for MSY consomic rats provides the first large-scale phenotyping for MSY contributions. Phenotypes were measured on consomic FHH-Y ${ }^{\mathrm{BN}} / \mathrm{Mcwi}$ (BN MSY introgressed onto $\mathrm{FHH}$ genomic background) and $\mathrm{SS}-\mathrm{Y}^{\mathrm{BN}} / \mathrm{Mcwi}$ (BN MSY introgressed onto SS genomic background). In FHH$\mathrm{Y}^{\mathrm{BN}} /$ Mcwi and SS-Y $\mathrm{Y}^{\mathrm{BN}} / \mathrm{Mcwi}, 131$ and 223 phenotypes respectively were measured. When comparing values for $\mathrm{FHH}-\mathrm{Y}^{\mathrm{BN}} / \mathrm{Mcwi}$ to male $\mathrm{FHH}$ rats, 28 phenotypes are significantly different (Additional file 1: Table S5). When comparing values for SS- $\mathrm{Y}^{\mathrm{BN}} / \mathrm{Mcwi}$ to male SS rats, 29 phenotypes are significantly different (Additional file 1: Table S6). As a percent of total phenotypes observed to be significantly different in the consomic relative to parental strain per megabase $(\mathrm{Mb})$ of DNA, MSY has a $36.09 \pm 3.84$-fold $(47.23 \pm 6.17$-fold in BNxFHH and $24.95 \pm 3.05$-fold in $\mathrm{BNxSS}$ ) higher contribution to phenotypes than any other chromosome consomic generated (Fig. 6a). Although chromosome size of the MSY differs between rat strains [12], there has yet been evidence of non-MSY sequence driving the changes in size; thus, the genes present in the sequenced SHR are also likely to drive the phenotypes altered by MSY consomics through either copy number variants or SNVs. This is the first evidence that many broad phenotypes in rat lab strains reflect, at least in part, MSY variation.

Additional analysis of MSY contribution can be observed by comparing male to female phenotypes for a particular strain. From the $28 \mathrm{FHH}-\mathrm{Y}^{\mathrm{BN}} / \mathrm{Mcwi}$ and 29 SS- $\mathrm{Y}^{\mathrm{BN}} / \mathrm{Mcwi}$ significant phenotypes, the difference between male to female parental animals (BN, FHH, and SS) was calculated for each phenotype (Table 3). Of the traits seen significantly altered in $\mathrm{FHH}-\mathrm{Y}^{\mathrm{BN}} / \mathrm{Mcwi}, 15$ phenotypes show a significant sex difference in both $\mathrm{FHH}$ and $\mathrm{BN}$, while only 2 phenotypes show FHH sex specific differences and 10 phenotypes show BN sex-specific differences (Fig. 6b and Table 3). Of the phenotypes seen significantly altered in SS- $\mathrm{Y}^{\mathrm{BN}} / \mathrm{Mcwi}, 22$ phenotypes show sex differences in both $\mathrm{SS}$ and $\mathrm{BN}$, while 1 phenotype shows SS sex-specific difference and 3 phenotypes show BN sex-specific differences (Fig. $6 \mathrm{~b}$ and Table 3).

There were two traits that overlapped in both MSY consomic rats that have a BN sex-specific difference, dilator response to acetylcholine EC50, and dilator response to acetylcholine Log EC50. With male BN rats having a higher response than female rats, it is surprising that FHH-Y ${ }^{\mathrm{BN}} / \mathrm{Mcwi}$ (Fig. 6c) and SS-Y ${ }^{\mathrm{BN}} / \mathrm{Mcwi}$ (Fig. 6d) significantly decrease the response relative to either FHH or SS. The large sex difference in the BN rat migrates only with chromosome 15 between the two consomic panels, FHH- $15^{\mathrm{BN}} / \mathrm{Mcwi}$ and $\mathrm{SS}-15^{\mathrm{BN}} / \mathrm{Mcwi}$, 


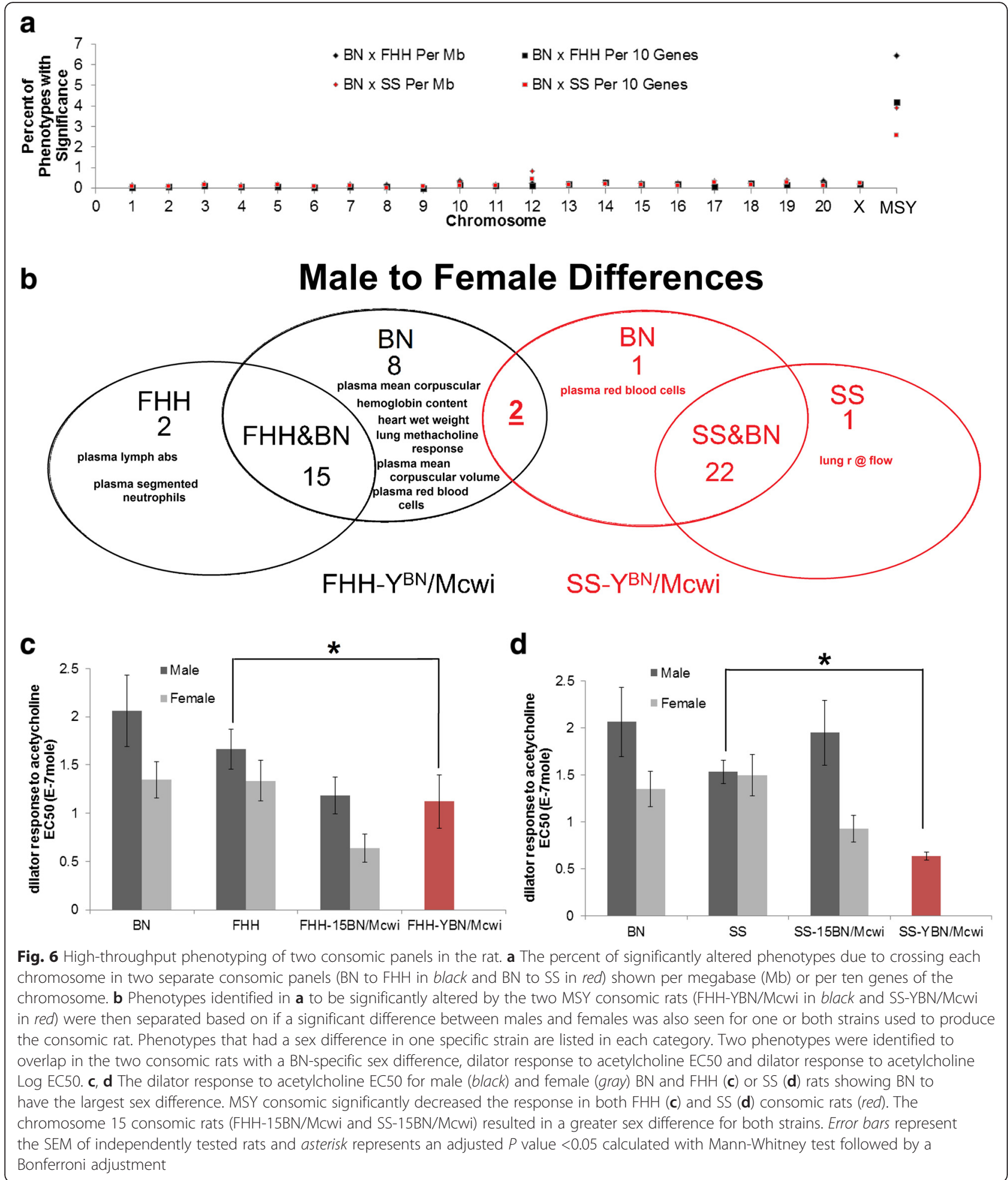

suggesting chromosome 15 is responsible for $\mathrm{BN}$ specific sex difference in acetylcholine response, but MSY may have evolved to partially attenuate this response in the BN rat (Fig. 6d, e).

\section{Discussion}

Few animal models currently exist to study the contributions of MSY to phenotypes, which could contribute to narrowing down genes that contribute to human disease. 
Table 3 MSY altered phenotypes with differences in male to female rat strains

\begin{tabular}{|c|c|c|c|c|c|}
\hline Phenotype & Rat treatment & $\begin{array}{l}\text { Consomic with } \\
\text { significant change }\end{array}$ & $\begin{array}{l}\text { Male to female } \\
\text { BN significant }\end{array}$ & $\begin{array}{l}\text { Male to female } \\
\text { FHH significant }\end{array}$ & $\begin{array}{l}\text { Male to female } \\
\text { SS significant }\end{array}$ \\
\hline Plasma lymph abs (E3) & $12 \% \mathrm{O}_{2}, 0.4 \%$ salt, male & FHH-YBN/Mcwi & No & Yes & - \\
\hline Plasma segmented neutrophils (E3) & $12 \% \mathrm{O}_{2}, 0.4 \%$ salt, male & $\mathrm{FHH}-\mathrm{YBN} / \mathrm{Mcwi}$ & No & Yes & - \\
\hline Plasma mean corpuscular hemoglobin content (pg) & $12 \% \mathrm{O}_{2}, 0.4 \%$ salt, male & $\mathrm{FHH}-\mathrm{YBN} / \mathrm{Mcwi}$ & Yes & No & - \\
\hline Plasma mean corpuscular volume (fL) & $12 \% \mathrm{O}_{2}, 0.4 \%$ salt, male & FHH-YBN/Mcwi & Yes & No & - \\
\hline Plasma red blood cell (E6/ $\mu \mathrm{L})$ & $21 \% \mathrm{O}_{2}, 0.4 \%$ salt, male & FHH-YBN/Mcwi & Yes & No & - \\
\hline Pre-ischemic heart wet weight (g) & $12 \% \mathrm{O}_{2}, 0.4 \%$ salt, male & FHH-YBN/Mcwi & Yes & No & - \\
\hline Pre-ischemic heart wet weight (g) & $21 \% \mathrm{O}_{2}, 0.4 \%$ salt, male & FHH-YBN/Mcwi & Yes & No & - \\
\hline Pre-ischemic left ventricle developed pressure $(\mathrm{mmHg})$ & $12 \% \mathrm{O}_{2}, 0.4 \%$ salt, male & FHH-YBN/Mcwi & Yes & No & - \\
\hline Pre-ischemic left ventricle systolic pressure $(\mathrm{mmHg})$ & $12 \% \mathrm{O}_{2}, 0.4 \%$ salt, male & FHH-YBN/Mcwi & Yes & No & - \\
\hline Methacholine ED50 (mg/kg) & $21 \% \mathrm{O}_{2}, 0.4 \%$ salt, male & FHH-YBN/Mcwi & Yes & No & - \\
\hline Dilator response to acetylcholine EC50 (E-7 mole) & $21 \% \mathrm{O}_{2}, 0.4 \%$ salt, male & FHH-YBN/Mcwi & Yes & No & - \\
\hline Dilator response to acetylcholine Log EC50 (Log molar) & $21 \% \mathrm{O}_{2}, 0.4 \%$ salt, male & FHH-YBN/Mcwi & Yes & No & - \\
\hline Plasma alk phos (U/L) & $21 \% \mathrm{O}_{2}, 0.4 \%$ salt, male & $\mathrm{FHH}-\mathrm{YBN} / \mathrm{Mcwi}$ & Yes & Yes & - \\
\hline Plasma globulin (g/dL) & $21 \% \mathrm{O}_{2}, 0.4 \%$ salt, male & $\mathrm{FHH}-\mathrm{YBN} / \mathrm{Mcwi}$ & Yes & Yes & - \\
\hline Plasma hematocrit (\%) & $21 \% \mathrm{O}_{2}, 0.4 \%$ salt, male & FHH-YBN/Mcwi & Yes & Yes & - \\
\hline Plasma hemoglobin (g/dL) & $21 \% \mathrm{O}_{2}, 0.4 \%$ salt, male & FHH-YBN/Mcwi & Yes & Yes & - \\
\hline Plasma phosphorus (mg/dL) & $21 \% \mathrm{O}_{2}, 0.4 \%$ salt, male & FHH-YBN/Mcwi & Yes & Yes & - \\
\hline Plasma total protein (g/dL) & $21 \% \mathrm{O}_{2}, 0.4 \%$ salt, male & FHH-YBN/Mcwi & Yes & Yes & - \\
\hline Plasma white blood cell count (E3/ $/ \mathrm{L})$ & $12 \% \mathrm{O}_{2}, 0.4 \%$ salt, male & FHH-YBN/Mcwi & Yes & Yes & - \\
\hline Ischemic peak contracture (mmHg) & $12 \% \mathrm{O}_{2}, 0.4 \%$ salt, male & FHH-YBN/Mcwi & Yes & Yes & - \\
\hline Body weight (kg) & $12 \% \mathrm{O}_{2}, 0.4 \%$ salt, male & FHH-YBN/Mcwi & Yes & Yes & - \\
\hline Body weight (kg) & $12 \% \mathrm{O}_{2}, 0.4 \%$ salt, male & FHH-YBN/Mcwi & Yes & Yes & - \\
\hline Body weight (kg) & $21 \% \mathrm{O}_{2}, 0.4 \%$ salt, male & FHH-YBN/Mcwi & Yes & Yes & - \\
\hline Lung dry wt (g)/body wt. (kg) ratio (g/kg) & $21 \% \mathrm{O}_{2}, 0.4 \%$ salt, male & FHH-YBN/Mcwi & Yes & Yes & - \\
\hline Hematocrit (\%) & $21 \% \mathrm{O}_{2}, 0.4 \%$ salt, male & $\mathrm{FHH}-\mathrm{YBN} / \mathrm{Mcwi}$ & Yes & Yes & - \\
\hline Rt ventricle/left ventricle weight ratio ( $w / w$ ratio) & $12 \% \mathrm{O}_{2}, 0.4 \%$ salt, male & FHH-YBN/Mcwi & Yes & Yes & - \\
\hline Body weight & $21 \% \mathrm{O}_{2}, 0.4 \%$ salt, male & $\mathrm{FHH}-\mathrm{YBN} / \mathrm{Mcwi}$ & Yes & Yes & - \\
\hline $\mathrm{r} @ \mathrm{flow}=100 \mathrm{ml} / \mathrm{min} / \mathrm{g}\left(\mathrm{mmHg} \times \min \times \mathrm{kg} \times \mathrm{ml}^{-1}\right)$ & $21 \% \mathrm{O}_{2}, 0.4 \%$ salt, male & SS-YBN/Mcwi & No & - & Yes \\
\hline 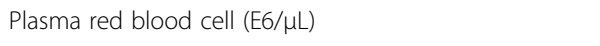 & $21 \% \mathrm{O}_{2}, 0.4 \%$ salt, male & SS-YBN/Mcwi & Yes & - & No \\
\hline Dilator response to acetylcholine EC50 ( $\mathrm{E}-7$ mole) & $21 \% \mathrm{O}_{2}, 0.4 \%$ salt, male & SS-YBN/Mcwi & Yes & - & No \\
\hline Dilator response to acetylcholine Log EC50 (Log molar) & $21 \% \mathrm{O}_{2}, 0.4 \%$ salt, male & SS-YBN/Mcwi & Yes & - & No \\
\hline Plasma calcium (mg/dL) & $21 \% \mathrm{O}_{2}, 0.4 \%$ salt, male & SS-YBN/Mcwi & Yes & - & Yes \\
\hline Plasma globulin (g/dL) & $21 \% \mathrm{O}_{2}, 0.4 \%$ Salt, Male & SS-YBN/Mcwi & Yes & - & Yes \\
\hline Plasma globulin (g/dL) & $12 \% \mathrm{O}_{2}, 0.4 \%$ salt, male & SS-YBN/Mcwi & Yes & - & Yes \\
\hline Plasma hematocrit (\%) & $12 \% \mathrm{O}_{2}, 0.4 \%$ salt, male & SS-YBN/Mcwi & Yes & - & Yes \\
\hline Plasma hemoglobin (g/dL) & $21 \% \mathrm{O}_{2}, 0.4 \%$ salt, male & SS-YBN/Mcwi & Yes & - & Yes \\
\hline Plasma hemoglobin ( $\mathrm{g} / \mathrm{dL}$ ) & $12 \% \mathrm{O}_{2}, 0.4 \%$ salt, male & SS-YBN/Mcwi & Yes & - & Yes \\
\hline Plasma mono abs (E3) & $21 \% \mathrm{O}_{2}, 0.4 \%$ salt, male & SS-YBN/Mcwi & Yes & - & Yes \\
\hline Plasma potassium (mmol/L) & $21 \% \mathrm{O}_{2}, 0.4 \%$ salt, male & SS-YBN/Mcwi & Yes & - & Yes \\
\hline 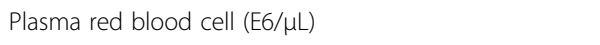 & $12 \% \mathrm{O}_{2}, 0.4 \%$ Salt, Male & SS-YBN/Mcwi & Yes & - & Yes \\
\hline Plasma total protein ( $\mathrm{g} / \mathrm{dL})$ & $12 \% \mathrm{O}_{2}, 0.4 \%$ salt, male & SS-YBN/Mcwi & Yes & - & Yes \\
\hline Ischemic time to onset of contracture (s) & $12 \% \mathrm{O}_{2}, 0.4 \%$ salt, male & SS-YBN/Mcwi & Yes & - & Yes \\
\hline Ischemic time to peak contracture (s) & $12 \% \mathrm{O}_{2}, 0.4 \%$ salt, male & SS-YBN/Mcwi & Yes & - & Yes \\
\hline Pre-ischemic heart rate (beats/min) & $12 \% \mathrm{O}_{2}, 0.4 \%$ salt, male & SS-YBN/Mcwi & Yes & - & Yes \\
\hline Pre-ischemic heart wet weight (g) & $12 \% \mathrm{O}_{2}, 0.4 \%$ salt, male & SS-YBN/Mcwi & Yes & - & Yes \\
\hline
\end{tabular}


Table 3 MSY altered phenotypes with differences in male to female rat strains (Continued)

\begin{tabular}{|c|c|c|c|c|c|}
\hline Body weight (kg) & $12 \% \mathrm{O}_{2}, 0.4 \%$ salt, male & SS-YBN/Mcwi & Yes & - & Yes \\
\hline Body weight (kg) & $12 \% \mathrm{O}_{2}, 0.4 \%$ salt, male & SS-YBN/Mcwi & Yes & - & Yes \\
\hline Lung dry wt (g)/body wt. (kg) ratio (g/kg) & $12 \% \mathrm{O}_{2}, 0.4 \%$ salt, male & SS-YBN/Mcwi & Yes & - & Yes \\
\hline Hematocrit (\%) & $21 \% \mathrm{O}_{2}, 0.4 \%$ salt, male & SS-YBN/Mcwi & Yes & - & Yes \\
\hline Hematocrit (\%) & $12 \% \mathrm{O}_{2}, 0.4 \%$ salt, male & SS-YBN/Mcwi & Yes & - & Yes \\
\hline Body weight (kg) & $21 \% \mathrm{O}_{2}, 0.4 \%$ salt, male & SS-YBN/Mcwi & Yes & - & Yes \\
\hline Rectal temperature after hypercapnia $\left({ }^{\circ} \mathrm{C}\right)$ & $21 \% \mathrm{O}_{2}, 0.4 \%$ salt, male & SS-YBN/Mcwi & Yes & - & Yes \\
\hline \% maximum relaxation acetylcholine (\%) & $21 \% \mathrm{O}_{2}, 0.4 \%$ salt, male & SS-YBN/Mcwi & Yes & - & Yes \\
\hline
\end{tabular}

Although many new tools are emerging to study human genetic-to-phenotype associations (such as iPS, organoid culturing, CRISPR/Cas9, and patient cell analysis), studying complex multi-tissue phenotypes and the ability to identify causal variants/genes to disease in a large genomic environment of thousands of inherited variants (such as the MSY) seen in the human still requires animal models. This paper set out to begin defining rat MSY genes in several commonly used rat strains to create models to study MSY variant contributions to phenotypes. The SHR MSY consomic rat (Fig. 1) has served as a model organism for studying the contribution of MSY genes, such as the SHR-specific Sry3 gene [28], in cardiovascular phenotypes. We have shown in this paper that the protein product of Sry3 can alter androgen receptor synergistic feedback and that delivery into the rat kidney results in pronounced blood pressure elevation that is blocked by a RAS inhibitor. This suggests a molecular mechanism into the repression of blood pressure response seen in the SHR/y cross to the Tfm rat, showing a synergistic response between MSY genetics and hormone signaling. Our identification of a relationship between genetic variation in rat Sry and blood pressure through the RAS promises to facilitate further studies of such a potential mechanistic link between human SRY and blood pressure regulation [22, 27].

Previous data for Sry in both rat and mouse suggest that the gene contributes to diverse phenotypes such as brain development [30] and that MSY genes in the mouse contribute to a number of phenotypes [3]; however, a large-scale phenotyping project has not yet been performed for any consomic animal strains before this paper. In this paper, we have shown that MSY per DNA base has a large phenotypic diversity between rat strains, a likely result of being a region within the genome to have uncontrolled mutations in inbred mating due to a lack of genetic crossover to remove de novo variants. This also highlights the importance of maintaining nomenclature on isolated breeding of strains, as MSY continues to diverge in separate mating locations and can result in different phenotypes based on mating location. This concept is supported by work in comparing the
SHR/Akr MSY consomic with the SHR/Crl consomic, which lacks the Sry3 duplication that results in blood pressure elevation [28] and thus lacks a blood pressure association [31]. This paper would also suggest a potential importance of mitochondrial genomics, as many of the inheritance patterns for that region would be under similar evolution as MSY in inbred animals; however, few studies have focused on this to date.

Furthermore, we have shown the expression profile for nine MSY genes found in rat and human. Using wholegenome sequencing reads from male rats, seven copies of Sry have been confirmed for the first time in multiple commonly used laboratory rat strains. The only Sry copy found ubiquitously expressed, Sry2, was inserted into an intron of the antisense strand of the ubiquitously expressed $K d m 5 d$ gene and also contains mutations damaging to nuclear localization and transcriptional regulation. With initial fragment analysis protocols only amplifying Sry2 in an orientation-dependent manner, without amplifying $K d m 5 d$ introns, the high expression of $\operatorname{Sry} 2$ is likely driven by global chromatin state around the $K d m 5 d$ gene and is not transcripts detected from $\mathrm{Kdm} 5 \mathrm{~d}$ intron background of RNAseq. This highlights the fact that the location of genomic insertion can drive an expression profile; however, instead of being able to select on the removal of the gene as would be done on autosomes, MSY sequence has likely been selected on to remove functionality of the protein, maintaining future bulk of MSY contrary to previous MSY degradation theories. Other functional Sry copies are found expressed in specific tissues including the testis, kidney, lung, and spleen, similar to the expression profile of human SRY. This suggests that "undifferentiated" Sry gene copy expression data (generated by techniques such as real-time PCR) biases the understanding of specific Sry copies, with the use of sequencing-based technologies or our fragment analysis protocol (can separate out Sry2 from other copies), a more reliable method for Sry expression analysis in future rat work.

The technique of identifying male-specific variants in duplicated genes, although utilized here only in rat, could be used in the future to identify additional 
mammalian species-specific duplications onto MSY. The assembly of MSYs from several mammals has elucidated duplication events such as CDY [32], FLJ36031Y [33], ZNF280AY [34], and MBTPS2Y [1]. However, with only a handful of sequenced MSYs in mammals, the extent of such gene duplications throughout mammalian evolution is unknown. The technique shown here is capable of identifying MSY duplications from reads of several male (or male-contaminated female samples) and female whole-genome sequences, a task that has become relatively inexpensive with next-generation sequencing technologies.

Gene duplication has been recognized as a driver of phenotypic changes for human diseases [35]. Studying duplication of autosomal genes onto MSY provides a unique opportunity to understand mechanisms and selective pressure of gene duplication, in addition to assessing the current status of our genomic scaffold. Of seven duplication events detected in our work, only two genes were found as expressed transcripts, with five becoming pseudogenes. Of the two genes that maintain expression, it is shown that Med14y was not present in rat strains such as FHL. Duplication of Ube2q2, a gene associated with kidney function [36], onto MSY was initially considered a pseudogene in SHR sequence annotation [2]. However, when analyzing the F344 sequence, transcripts were found ubiquitously expressed from MSY (using the male-specific SNVs). Analysis of 197 male RNAseq datasets from the rat identified 35 additional RNAseq datasets to have detectable Ube $2 q 2 Y$ transcripts, suggesting strain-specific stratification in expression. The repetitive nature of rat MSY has made it challenging to generate a complete MSY sequence for rat. The analysis of Sry and autosomal gene duplications on MSY can serve as markers for completeness of our current assemblies. For example, the SNVs found in Sry3C or pseudogenes Ect $2 Y_{-} p s$, Havcr2Y_ps, Prrc2cY_ps, and Vom $2 r Y_{-} p s$, which have been confirmed in multiple male rat genomes, are not found in the current Rnor 6.0 MSY assembly. These therefore serve as valuable markers in the future for completing a rat MSY sequence.

\section{Conclusions}

Tools to genetically modify rat strains are rapidly increasing in use [37], allowing for the assessment of a single-gene modification in the vast array of genetic landscapes [15] present in rat research. Identification and characterization of rat MSY genes in this paper opens the door for rat MSY gene editing to study sex differences in diseases. Hopefully, this approach can narrow down the large haplotype block of human MSY to specific genes that contribute to disease association and also suggest approaches that can be used for other species (mouse and primates) to study future MSY phenotype contributions. We now have a causal relationship established in the SHR MSY for the Sry gene to cardiovascular disease, allowing for focus on human variants in MSY haplotype groups associated with cardiovascular disease to the $S R Y$ gene regions, while also implicating AR signaling to influence blood pressure control through SRY synergistic transcriptional regulation.

Utilizing a new male/female SNV segregating approach based on whole-genome sequence reads, we have shown a promising new technique in identifying gene duplications onto MSY that may be critical in identifying species-specific duplication events. Two (Med14Y and Ube2q2Y) functional MSY retroposed genes (out of ten duplication events shared in most rat strains analyzed) are shown in this paper to have strain-specific variation. The strain-specific outcomes of these duplication events and the high mutation rate of MSY in inbred rat populations suggest a major concern, particularly in light of the phenotypic role MSY is shown to have in this paper. Two consomic panels of inbred rats show MSY to contribute 36-fold more per chromosome size to inbred strain phenotypes than any other chromosome in the rat genome. The combination of approaches taken in this paper to analyze rat MSY genes highlights the importance of MSY to phenotype/disease, suggesting inbred models such as rat are ideal to dissect mechanisms of human MSY genes involved in sex differences. Once gene-to-phenotype relationships are established for these animal models, we envisage that the research community might exploit CRISPR/Cas9 modification of human cell lines to investigate relationships between human MSY variants to disease states.

\section{Additional file}

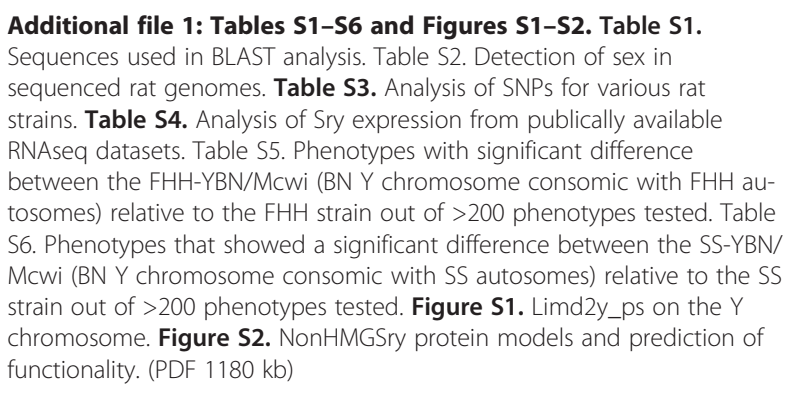

\section{Abbreviations}

AR: Androgen receptor; MSY: Male-specific region of the $Y$ chromosome; RAS: Renin-angiotensin system.

\section{Competing interests}

The authors declare that they have no competing interests.

\section{Authors' contributions}

JWP performed Sry genomic analyses over rat strains, RNAseq data analysis, and protein modeling. SWT and ABF performed variant analysis for rat strains. SB and DE performed and interpreted Sry3 rat blood pressure studies. 
ACU performed Sry2 functional studies. ST performed assays for SRY-AR synergistic regulation. LP, AM, MET, and ASM performed and interpreted expression analysis of Sry. MT organized and developed the RN6.0 variant visualizer tool. JWP, JL, HJJ, and MRD advised and oversaw project completion. JWP wrote the manuscript and all authors approved its submission.

\section{Acknowledgements}

We thank the Human Genome Sequencing Center at Baylor College of Medicine for the public database deposits of finished rat MSY BAC sequences. We thank Helen Skaletsky, Jennifer Hughes, and David Page of the Whitehead Institute, Howard Hughes Medical Institute, and Massachusetts Institute of Technology for providing a list of SHR identified genes and help with the analysis of SHR sequencing BACs. In addition, we thank Dr. Leming Shi of Fudan University for the approval and help in utilizing the Rat BodyMap dataset. The sequence for Ube2q2Y is deposited in GenBank (KM610331). This work was supported by the National Institutes of Health (grant number R01 HL071579 to D.E., R01 HL069321 to H.J., K01 ES025435 to J.W.P.) and the American Heart Association (PRE 7380033 to J.W.P.).

\section{Author details}

${ }^{1}$ HudsonAlpha Institute for Biotechnology, 601 Genome Way, Huntsville, AL 35806, USA. ${ }^{2}$ Human and Molecular Genetics Center, Medical College of Wisconsin, Milwaukee, WI 53226, USA. ${ }^{3}$ Department of Physiology, Medical College of Wisconsin, Milwaukee, WI 53226, USA. ${ }^{4}$ Department of Biology, The University of Akron, Akron, $\mathrm{OH} 44325$, USA. ${ }^{5}$ Department of Mathematics and Science, Walsh University, North Canton, OH 44720, USA. ${ }^{6}$ Núcleo de Fisiologia Geral e Genômica Funcional-ICB-Universidade Federal de Minas Gerais (UFMG), Belo Horizonte, Minas Gerais, Brazil.

Received: 4 November 2015 Accepted: 26 January 2016 Published online: 03 February 2016

\section{References}

1. Cortez D, Marin R, Toledo-Flores D, Froidevaux L, Liechti A, Waters PD, et al. Origins and functional evolution of $Y$ chromosomes across mammals. Nature. 2014:508:488-93.

2. Bellott DW, Hughes JF, Skaletsky H, Brown LG, Pyntikova T, Cho T-J, et al. Mammalian Y chromosomes retain widely expressed dosage-sensitive regulators. Nature. 2014;508:494-9.

3. Prokop JW, Deschepper CF. Chromosome Y genetic variants: impact in animal models and on human disease. Physiol Genomics. 2015: physiolgenomics.00074.2015.

4. Forsberg LA, Rasi C, Malmqvist N, Davies H, Pasupulati S, Pakalapati G, et al. Mosaic loss of chromosome $Y$ in peripheral blood is associated with shorter survival and higher risk of cancer. Nat Genet. 2014:46:624-8.

5. Ober C, Loisel DA, Gilad Y. Sex-specific genetic architecture of human disease. Nat Rev Genet. 2008:9:911-22.

6. Rossouw JE. Hormones, genetic factors, and gender differences in cardiovascular disease. Cardiovasc Res. 2002;53:550-7.

7. Bloomer LDS, Nelson CP, Eales J, Denniff M, Christofidou P, Debiec R, et al. Male-specific region of the $Y$ chromosome and cardiovascular risk: phylogenetic analysis and gene expression studies. Arterioscler Thromb Vasc Biol. 2013;33:1722-7.

8. Charchar FJ, Bloomer LD, Barnes TA, Cowley MJ, Nelson CP, Wang Y, et al, Inheritance of coronary artery disease in men: an analysis of the role of the Y chromosome. Lancet. 2012;379:915-22.

9. Ely DL, Turner ME. Hypertension in the spontaneously hypertensive rat is linked to the Y chromosome. Hypertension. 1990;16:277-81.

10. Ely DL, Daneshvar H, Turner ME, Johnson ML, Salisbury RL. The hypertensive Y chromosome elevates blood pressure in F11 normotensive rats. Hypertension. 1993;21(6 Pt 2):1071-5.

11. Davidson AO, Schork N, Jaques BC, Kelman AW, Sutcliffe RG, Reid JL, et al. Blood pressure in genetically hypertensive rats. Influence of the $Y$ chromosome. Hypertension. 1995;26:452-9.

12. Kren V, Qi N, Krenova D, Zidek V, Sladká M, Jáchymová M, et al. Y-chromosome transfer induces changes in blood pressure and blood lipids in SHR. Hypertension. 2001;37:1147-52.

13. Mattson DL, Dwinell MR, Greene AS, Kwitek AE, Roman RJ, Cowley AW, et al. Chromosomal mapping of the genetic basis of hypertension and renal disease in FHH rats. Am J Physiol Renal Physiol. 2007;293:F1905-1914.
14. Mattson DL, Dwinell MR, Greene AS, Kwitek AE, Roman RJ, Jacob HJ, et al. Chromosome substitution reveals the genetic basis of Dahl salt-sensitive hypertension and renal disease. Am J Physiol Renal Physiol. 2008;295:F837-842.

15. Atanur SS, Diaz AG, Maratou K, Sarkis A, Rotival M, Game L, et al. Genome sequencing reveals loci under artificial selection that underlie disease phenotypes in the laboratory rat. Cell. 2013;154:691-703.

16. Yu Y, Fuscoe JC, Zhao C, Guo C, Jia M, Qing T, et al. A rat RNA-Seq transcriptomic BodyMap across 11 organs and 4 developmental stages. Nat Commun. 2014;5:3230.

17. Turner M, Martin C, Martins A, Dunmire J, Farkas J, Ely D, et al. Genomic and expression analysis of multiple Sry loci from a single Rattus norvegicus $Y$ chromosome. BMC Genet. 2007;8:11.

18. Xu D, Zhang Y. Ab initio protein structure assembly using continuous structure fragments and optimized knowledge-based force field. Proteins. 2012:80:1715-35.

19. Duan Y, Wu C, Chowdhury S, Lee MC, Xiong G, Zhang W, et al. A pointcharge force field for molecular mechanics simulations of proteins based on condensed-phase quantum mechanical calculations. J Comput Chem. 2003;24:1999-2012.

20. Dinkel H, Michael S, Weatheritt RJ, Davey NE, Roey KV, Altenberg B, et al. ELM-the database of eukaryotic linear motifs. Nucleic Acids Res. 2011:gkr1064.

21. Prokop JW, Underwood AC, Turner ME, Miller N, Pietrzak D, Scott S, et al. Analysis of Sry duplications on the Rattus norvegicus Y-chromosome. BMC Genomics. 2013;14:792.

22. Prokop JW, Watanabe IKM, Turner ME, Underwood AC, Martins AS, Milsted A. From rat to human: regulation of renin-angiotensin system genes by sry. Int J Hypertens. 2012;2012:724240.

23. Ely D, Boehme S, Dunphy G, Hart M, Chiarappa F, Miller B, et al. The Sry3 Y chromosome locus elevates blood pressure and renin-angiotensin system indexes. Gend Med. 2011;8:126-38.

24. Kwitek AE, Jacob HJ, Baker JE, Dwinell MR, Forster HV, Greene AS, et al. BN phenome: detailed characterization of the cardiovascular, renal, and pulmonary systems of the sequenced rat. Physiol Genomics. 2006;25:303-13.

25. Peng H, Talebzadeh-Farrooji M, Osborne MJ, Prokop JW, McDonald PC, Karar J, et al. LIMD2 is a small LIM-only protein overexpressed in metastatic lesions that regulates cell motility and tumor progression by directly binding to and activating the integrin-linked kinase. Cancer Res. 2014;74:1390-403.

26. Chang BH, Shimmin LC, Shyue SK, Hewett-Emmett D, Li WH. Weak maledriven molecular evolution in rodents. Proc Natl Acad Sci U S A. 1994;91:827-31.

27. Araujo FC, Milsted A, Watanabe IKM, Puerto HLD, Santos RAS, Lazar J, et al. Similarities and differences of $X$ and $Y$ chromosome homologous genes, SRY and SOX3, in regulating the renin-angiotensin system promoters. Physiol Genomics. 2015:physiolgenomics.00138.2014.

28. Turner ME, Farkas J, Dunmire J, Ely D, Milsted A. Which Sry locus is the hypertensive Y chromosome locus? Hypertension. 2008;53:430-5.

29. Yuan X, Lu ML, Li T, Balk SP. SRY interacts with and negatively regulates androgen receptor transcriptional activity. J Biol Chem. 2001;276:46647-54.

30. Turner ME, Ely D, Prokop J, Milsted A. Sry, more than testis determination? Am J Physiol Regul Integr Comp Physiol. 2011;301(3):R561-571.

31. Vincent M, Kaiser MA, Orea V, Lodwick D, Samani NJ. Hypertension in the spontaneously hypertensive rat and the sex chromosomes. Hypertension. 1994;23:161-6.

32. Lahn BT, Page DC. Retroposition of autosomal mRNA yielded testis-specific gene family on human Y chromosome. Nat Genet. 1999;21:429-33.

33. Murphy WJ, Pearks Wilkerson AJ, Raudsepp T, Agarwala R, Schäffer AA, Stanyon $\mathrm{R}$, et al. Novel gene acquisition on carnivore $\mathrm{Y}$ chromosomes. PLoS Genet. 2006;2:e43

34. Yang Y, Chang T-C, Yasue H, Bharti AK, Retzel EF, Liu W-S. ZNF280BY and ZNF280AY: autosome derived Y-chromosome gene families in Bovidae. BMC Genomics. 2011;12:13.

35. Conrad B, Antonarakis SE. Gene duplication: a drive for phenotypic diversity and cause of human disease. Annu Rev Genomics Hum Genet. 2007:8:17-35.

36. Köttgen A, Pattaro C, Böger CA, Fuchsberger C, Olden M, Glazer NL, et al. New loci associated with kidney function and chronic kidney disease. Nat Genet. 2010;42:376-84.

37. Flister MJ, Prokop JW, Lazar J, Shimoyama M, Dwinell M, Geurts A. 2015 guidelines for establishing genetically modified rat models for cardiovascular research. J Cardiovasc Transl Res. 2015;8:269-77. 